\title{
Decision Support System For Determining the Best College High Private Using Topsis Method
}

\author{
Yuyun Dwi Lestari \\ Universitas Harapan Medan \\ Medan, Indonesia \\ yuyun.dl@gmail.com
}

\author{
Mardiana \\ Universitas Harapan Medan \\ Medan, Indonesia \\ mardiana.mcom@gmail.com
}

Submitted: Oct 3, 2019

Accepted: Mar 15, 2020

Published: Apr 1, 2020

\begin{abstract}
In this study, the Best Private Universities in Medan will be discussed with the TOPSIS method (Techniques for Other References with Similarities to Solutions). TOPSIS is one method of Decision Support Systems that is used to accept decisions that have many criteria. The general objective of this study is to use the TOPSIS method which is expected to be able to provide optimal results in determining the best universities. And specific targets of this study can provide estimates or indicators that can be used by Universities in Medan in determining whether the College is the best or not. The criteria used in this study were Institutional, Student Activities, Lecturer HR, Research and Community Service, and Innovation. In the future these criteria can be added according to the needs and requirements provided by the Ministry of Research, Technology and Higher Education. By applying the TOPSIS Method to Decision Supporters in the selection of the Best Private Universities in Medan City can provide optimal results based on the criteria and weighting that has been determined.
\end{abstract}

\section{Keywords-Decision Support System, TOPSIS, Higher Education, Ranking}

\section{INTRODUCTION}

Ranking of a tertiary institution, both state and private tertiary institutions, can be a reference for these tertiary institutions to attract students. The higher the rank of a tertiary institution, the more popular the campus is for prospective students to enter. To determine the ranking of a campus or university a cluster mapping scheme is needed under the auspices of the Ministry of Research, Technology and Higher Education to improve the quality of universities on a regular and sustainable basis. Quoted from the page ristekdikti.go.id there are 5 assessment components that are the basis for ranking universities under the auspices of the Ministry of Research and Technology including Human Resources Quality, Institutional Quality, Student Activity Quality, Research and Community Service Quality, and Innovation Quality (Kemenristekdikti, 2018) .

This research will create a Decision Support System to determine the best private universities by using the TOPSIS (Technique for Order of Preference by Similarity to Ideal Solution) method. The number of private universities in Medan by offering their respective advantages will make prospective students interested in getting into which private universities are the best. Therefore, each tertiary institution needs to adopt any indicators that are an assessment for students so that they can be interested in entering the tertiary institution.

TOPSIS has been used in many applications including financial investment decisions, company performance comparison, comparison in a specific industry, operating system selection, customer evaluation, and robot. (Muzakkir, 2017)

TOPSIS is a method for finding the ideal solution based on the value of preference. The reason for using the TOPSIS method is that in TOPSIS it uses the concept of selected alternatives not only to have the shortest distance from the positive ideal solution, but also to have the longest distance from the negative ideal solution. The concept of TOPSIS is simple and easy to understand and has the ability to measure 
alternative alternatives in mathematical form. (Firdaus, Abdillah, \& Renaldi, 2016).

Research conducted by (Erik Kurniawan, Hindayati Mustafidah, 2015) with the title TOPSIS Method for Determining Admission of New Medical Education Students at the University of Muhammadiyah Purwakerto, in the study that the highest value of new prospective students can be taken into consideration in the process of selecting new student admissions at the Faculty of Medicine Muhammadiyah University. After conducting research into new student admissions, further research can determine student achievement. This research was conducted by (Herawatie \& Wuryanto, 2017).

Other research conducted by (Saleh \& Information, 2016) this study determines the majors for students conducted by the student section where the assessment process refers to report cards, written test results, and students' interests which are then recapitulated and then calculated. Next (Santiary, Ciptayani, Saptariani, \& I Ketut Swardika, 2018) by determining tourist sites in the city of Bali with the TOPSIS method. There are also researchers who conduct research using the AHP and TOPSIS methods for smartphone brand selection. (Akmaludin \& Badrul, 2019)

\section{LITERATURE REVIEW}

\subsection{Decision Support System}

Decision support system is an interactive computer-based information system, by processing data with various models to solve unstructured problems so that it can provide information that can be used by decision makers in making a decision. Decision making is a process of choosing an action among several alternatives, so that the desired objectives can be achieved. (Chamid, 2016)

The objectives of the decision support system are as follows: (Badrul, Rusdiansyah \& Budihartanti, 2019)

1. Assist in making decisions on structured problems

2. Providing support for the manager's consideration and not intended to replace the manager's function

3. Increasing the effectiveness of decisions taken more than improving efficiency.

4. The speed of computer computing enables decision makers to do a lot of computing quickly at a low cost

5. Increased productivity building a decisionmaking group, especially experts, can be very expensive
2.2 Technique For Order Preference By Similarity To Ideal Solution (TOPSIS)

The TOPSIS method is widely used to complete practical decision making. This is because the TOPSIS method has a simple and easy to understand concept, with efficient computing, and has the ability to measure the relative performance of decision alternatives in a simple mathematical form. In general, the TOPSIS method process follows the following steps (Kusumadewi, 2006)

1. Make a normalized decision matrix.

2. Make a normalized weighted decision matrix.

3. Determine a positive ideal solution matrix and a negative ideal solution matrix.

4. Determine the distance between the values of each alternative with a positive and negative ideal solution matrix.

5. Determine the preference value for each alternative.

TOPSIS method is also widely used to solve practical decision problems and the concept is simple, easy to understand, efficient computation and has the ability to measure the relative performance of decision alternatives in a simple mathematical form (Wahyuni Industry, Khairunnisa, Abriyani, Muchlis, \& Ulfa, 2017).

\section{Proposed Method}

\subsection{Research Subject}

The subjects in this study were campuses in the city of Medan to be ranked. The object of research is the ranking or ranking values of the campuses based on the criteria used. The variables used as rating criteria are:

\section{HR Quality}

2. Institutional Quality

3. Quality of Student Activities

4. Quality of Research and Community Service

5. Quality of Innovation

\subsection{Data Used}

In this study the data used to support the success of the study are as follows:

1. Campus or College Data in Medan City, 
2. HR data such as the number of lecturers based on education and comparison of the percentage of the number of students.

3. Institutional Data such as Accreditation.

4. Student Activity Data owned by the college.

5. Research and Community Service Data based on cluster, and scientific publication data based on rank in Sinta2.

6. Innovation data owned by tertiary institutions is based on Ristekdikti innovation data.

\subsection{Analysis of Method Implementation}

This section will explain how the TOPSIS method is applied in the selection of the best tertiary institution in Medan. The first stage determines the criteria and weight of each criterion in which the criteria used are:

a. $\mathrm{C} 1=\mathrm{HR}$ : Percentage of Lecturers and Students
a. $1: 15-1: 20 \quad: 4$
b. $1: 21-1: 25 \quad: 3$
c. $1: 26-1: 35 \quad: 2$
d. $<15$ or $>35 \quad: 1$

b. $\quad \mathrm{C} 2=\mathrm{HR}$ : Lecturer with Bechelor Degree Education (Percentage)
a. $0 \%$
$: 4$
b. $0.1 \%-0.99 \%$
$: 3$
c. $1 \%-8 \%$
$: 2$
d. $>8 \%$
$: 1$

c. $\mathrm{C} 3=\mathrm{HR}$ : Lecturer with Magister Degree Education (Percentage)
a. $80 \%-100 \%$
$: 4$
b. $60 \%-79.99 \%$
$: 3$
c. $40 \%-59.99 \%: 2$
d. $0 \%-39.99 \%: 1$

d. $\quad \mathrm{C} 4=\mathrm{HR}$ : Lecturer with Doctoral Degree Education (Percentage)
a. $10 \%-15 \%$
$: 4$
b. $7 \%-9.99 \%$
$: 3$
c. $3 \%-6.99 \%$
d. $0 \%-2.99 \%$
$: 2$
$: 1$

e. $\quad \mathrm{C} 5=$ Institutional: Accreditation
a. A
$: 4$
b. B
$: 3$
c. $\mathrm{C}$
$: 2$
d. -
$: 1$

f. $\quad$ C6 $=$ Student Activities
a. $\quad>3.00$
$: 4$
b. $\quad 1.00-2.99$
$: 3$
c. $0.1-0.99$
d. 0
$: 2$
$: 1$
a. Mandiri
$: 4$
b. Utama
c. Madya
$: 3$
d. Binaan
$: 2$

h. $\mathrm{C} 8=\mathrm{RCS}$ : Community Services
a. Unggul : 4
b. Sangat Bagus : 3
c. Memuaskan : 2
d. Kurang Memuaskan : 1

i. $\quad$ C9 = RCS: Publication (Sinta Ratings)
a. $1-300$ $: 4$
b. $301-500$
$: 3$
c. $501-700$
$: 2$
d. $>701$
$: 1$

j. $\quad \mathrm{C} 10=$ Inovation (Number of Inovation)
a. $>13$
$: 4$
b. $8-12$
c. $4-7$
d. $0-3$
$: 3$
$: 2$
$: 1$

1. The second stage determines the preference weights of each criterion, is:

a. $\mathrm{C} 1=\mathrm{HR}$ : Percentage of Lecturers and Students = 3

b. $\mathrm{C} 2=\mathrm{HR}$ : Lecturer with Bechelor Degree Education (Percentage) $=3$

c. C3 = HR: Lecturer with Magister Degree Education (Percentage) $=3$

d. C4 = HR: Lecturer with Doctoral Degree Education (Percentage) $=3$

e. $\mathrm{C} 5=$ Institutional: Accreditation $=4$

f. $\mathrm{C} 6=$ Student Activities $=1$

g. $\mathrm{C7}=\mathrm{RCS}:$ Research $=3$

h. $\mathrm{C} 8=\mathrm{RCS}$ : Community Services $=3$

i. $\quad$ C9 = RCS: Publication (Sinta Rating) $=3$

j. $\quad$ C10 $=$ Inovation $($ Number of Inovation $)=1$

\section{RESUlt AND DiscuSSION}

At this stage will explain the results achieved from the application of the TOPSIS method to the data to be processed. These data will be used as criteria in the calculation process. Data obtained and processed are as follows:

g. $\quad$ C7 = RCS: Research 
TABLE I.

Data ObTained AND WiLl BE PROCESSED

\begin{tabular}{|c|c|c|c|c|c|}
\hline \multirow[b]{2}{*}{ No } & \multirow[b]{2}{*}{$\begin{array}{c}\text { Perguruan } \\
\text { Tinggi }\end{array}$} & \multicolumn{4}{|c|}{ SDM } \\
\hline & & $\begin{array}{c}\text { Persentase Dosen } \\
\text { Mahasiswa } \\
(1: \ldots)\end{array}$ & Dosen S1 & $\begin{array}{c}\text { Dosen } \\
\text { S2 }\end{array}$ & Dosen S3 \\
\hline 1 & A & 50.8 & 1 & 201 & 32 \\
\hline 2 & B & 42 & 18 & 450 & 70 \\
\hline 3 & $\mathrm{C}$ & 15 & 8 & 293 & 41 \\
\hline 4 & $\mathrm{D}$ & 17.7 & 14 & 166 & 7 \\
\hline 5 & E & 33.4 & 9 & 148 & 13 \\
\hline 6 & $\mathrm{~F}$ & 64.6 & 4 & 86 & 14 \\
\hline 7 & G & 45 & 10 & 289 & 32 \\
\hline 8 & $\mathrm{H}$ & 37.7 & 46 & 265 & 50 \\
\hline 9 & I & 34 & 2 & 223 & 25 \\
\hline 10 & $\mathrm{~J}$ & 1.1 & 3 & 57 & 1 \\
\hline \multirow{2}{*}{ No } & \multirow{2}{*}{$\begin{array}{c}\text { Perguruan } \\
\text { Tinggi }\end{array}$} & \multicolumn{4}{|c|}{ Kelembagaan } \\
\hline & & \multicolumn{4}{|c|}{ Akreditasi Perguruan Tinggi } \\
\hline 1 & $\mathrm{~A}$ & \multicolumn{4}{|c|}{$\mathrm{B}$} \\
\hline 2 & B & \multicolumn{4}{|c|}{$\mathrm{A}$} \\
\hline 3 & $\mathrm{C}$ & \multicolumn{4}{|c|}{ В } \\
\hline 4 & $\mathrm{D}$ & \multicolumn{4}{|c|}{$\mathrm{C}$} \\
\hline 5 & E & \multicolumn{4}{|c|}{ B } \\
\hline 6 & $\mathrm{~F}$ & \multicolumn{4}{|c|}{$\mathrm{C}$} \\
\hline 7 & G & \multicolumn{4}{|c|}{ B } \\
\hline 8 & $\mathrm{H}$ & \multicolumn{4}{|c|}{ B } \\
\hline 9 & I & \multicolumn{4}{|c|}{ B } \\
\hline 10 & $\mathrm{~J}$ & \multicolumn{4}{|c|}{-} \\
\hline \multirow[b]{2}{*}{ No } & \multirow{2}{*}{$\begin{array}{l}\text { Perguruan } \\
\text { Tinggi }\end{array}$} & \multicolumn{4}{|c|}{ Kemahasiswaan } \\
\hline & & \multicolumn{4}{|c|}{$\begin{array}{c}\text { Nilai (Berdasarkan Nilai pada } \\
\text { pemeringkatan.ristekdikti.go.id) }\end{array}$} \\
\hline 1 & A & \multicolumn{4}{|c|}{0.074} \\
\hline 2 & B & \multicolumn{4}{|c|}{0.708} \\
\hline 3 & $\mathrm{C}$ & & 0 & & \\
\hline 4 & $\mathrm{D}$ & & 0.226 & & \\
\hline 5 & E & & 0.163 & & \\
\hline 6 & $\mathrm{~F}$ & & 0.034 & & \\
\hline 7 & G & & 0.129 & & \\
\hline 8 & $\mathrm{H}$ & & 0 & & \\
\hline 9 & I & & 0.094 & & \\
\hline 10 & $\mathrm{~J}$ & & 0 & & \\
\hline & & Peneliti: & Pengabdian & Publik & \\
\hline No & $\begin{array}{c}\text { Perguruan } \\
\text { Tinggi }\end{array}$ & Penelitian & $\begin{array}{r}\text { Pengabc } \\
\text { Masy }\end{array}$ & $\begin{array}{l}\text { n pada } \\
\text { akat }\end{array}$ & $\begin{array}{l}\text { Publikasi } \\
\text { (Sinta } \\
\text { Ranking) } \\
\end{array}$ \\
\hline 1 & A & Madya & Mem & skan & 288 \\
\hline 2 & B & Utama & Sanga & agus & 231 \\
\hline 3 & $\mathrm{C}$ & Madya & Mem & skan & 110 \\
\hline 4 & D & Madya & Mem & skan & 346 \\
\hline 5 & E & Binaan & Mem & skan & 411 \\
\hline 6 & $\mathrm{~F}$ & Madya & Mem & skan & 362 \\
\hline 7 & G & Madya & Mem & skan & 214 \\
\hline 8 & $\mathrm{H}$ & Binaan & $\begin{array}{r}\mathrm{Ku} \\
\mathrm{Mem} \\
\end{array}$ & & 195 \\
\hline 9 & I & Madya & Mem & skan & 242 \\
\hline
\end{tabular}

\begin{tabular}{|c|c|c|c|c|}
\hline 10 & $\mathrm{~J}$ & Binaan & $\begin{array}{c}\text { Kurang } \\
\text { Memuaskan }\end{array}$ & 1302 \\
\hline \multirow{2}{*}{ No } & \multirow{2}{*}{$\begin{array}{c}\text { Perguruan } \\
\text { Tinggi }\end{array}$} & \multicolumn{3}{|c|}{ Inovasi } \\
\hline & & \multicolumn{3}{|c|}{ Nilai (Berdasarkan data.inovasi.ristekdikti.go.id) } \\
\hline 1 & A & & 0 & \\
\hline 2 & B & & 0 & \\
\hline 3 & $\mathrm{C}$ & & 0 & \\
\hline 4 & D & & 0 & \\
\hline 5 & E & & 0 & \\
\hline 6 & $\mathrm{~F}$ & & 0 & \\
\hline 7 & $\mathrm{G}$ & & 0 & \\
\hline 8 & $\mathrm{H}$ & & 0 & \\
\hline 9 & I & & 0 & \\
\hline 10 & $\mathrm{~J}$ & & 0 & \\
\hline
\end{tabular}

From the data above we get the weighting matrix results from the alternative data held as follows:

\section{TABLE II. WEIGHTING ALL CRITERIA RESUlTS}

\begin{tabular}{|r|r|r|r|r|r|r|r|r|r|r|r|}
\hline No & $\begin{array}{c}\text { Data } \\
\text { Alternatif }\end{array}$ & $\mathrm{C} 1$ & $\mathrm{C} 2$ & $\mathrm{C} 3$ & $\mathrm{C} 4$ & $\mathrm{C} 5$ & $\mathrm{C} 6$ & $\mathrm{C} 7$ & $\mathrm{C} 8$ & $\mathrm{C} 9$ & $\mathrm{C} 10$ \\
\hline 1 & $\mathrm{~A} 1$ & 1 & 3 & 4 & 4 & 3 & 2 & 2 & 2 & 4 & 1 \\
\hline 2 & $\mathrm{~A} 2$ & 1 & 2 & 4 & 4 & 4 & 2 & 3 & 3 & 4 & 1 \\
\hline 3 & $\mathrm{~A} 3$ & 4 & 2 & 4 & 4 & 3 & 1 & 2 & 2 & 4 & 1 \\
\hline 4 & $\mathrm{~A} 4$ & 4 & 2 & 4 & 2 & 2 & 2 & 2 & 2 & 3 & 1 \\
\hline 5 & $\mathrm{~A} 5$ & 2 & 2 & 4 & 3 & 3 & 2 & 1 & 2 & 3 & 1 \\
\hline 6 & $\mathrm{~A} 6$ & 1 & 2 & 4 & 4 & 2 & 2 & 2 & 2 & 3 & 1 \\
\hline 7 & $\mathrm{~A} 7$ & 1 & 2 & 4 & 3 & 3 & 2 & 2 & 2 & 4 & 1 \\
\hline 8 & $\mathrm{~A} 8$ & 1 & 1 & 3 & 4 & 3 & 1 & 1 & 1 & 4 & 1 \\
\hline 9 & $\mathrm{~A} 9$ & 2 & 3 & 4 & 4 & 3 & 2 & 2 & 2 & 4 & 1 \\
\hline 10 & $\mathrm{~A} 10$ & 1 & 2 & 4 & 1 & 1 & 1 & 1 & 1 & 1 & 1 \\
\hline
\end{tabular}

After getting the weighting matrix results all the next step criteria create a matrix xij consisting of $\mathrm{m}$ alternatives and $\mathrm{n}$ criteria. This matrix contains the weights / grade of each alternative to each of the existing criteria:

\section{TABLE III. CRITERIA DATA}

\begin{tabular}{|c|c|c|}
\hline No & Kriteria & Weight \\
\hline 1 & C1 & 0,5 \\
\hline 2 & C2 & 0,5 \\
\hline 3 & C3 & 0,5 \\
\hline 4 & C4 & 0,5 \\
\hline 5 & C5 & 1 \\
\hline 6 & C6 & 0,25 \\
\hline 7 & C7 & 0,75 \\
\hline 8 & C8 & 0,75 \\
\hline 9 & C9 & 0,75 \\
\hline 10 & C10 & 0,25 \\
\hline
\end{tabular}


Next, calculate the weight of each data used:

TABLE IV. NORMALIZATION MATRIX RESUlTS

\begin{tabular}{|c|c|c|c|c|c|c|c|c|c|c|c|}
\hline No & $\begin{array}{c}\text { Data } \\
\text { Alternatif }\end{array}$ & $\mathrm{C} 1$ & $\mathrm{C} 2$ & $\mathrm{C} 3$ & $\mathrm{C} 4$ & $\mathrm{C} 5$ & $\mathrm{C} 6$ & $\mathrm{C} 7$ & $\mathrm{C} 8$ & $\mathrm{C} 9$ & C10 \\
\hline 1 & $\mathrm{~A} 1$ & 1 & 3 & 4 & 4 & 3 & 2 & 2 & 2 & 4 & 1 \\
\hline 2 & $\mathrm{~A} 2$ & 1 & 2 & 4 & 4 & 4 & 2 & 3 & 3 & 4 & 1 \\
\hline 3 & $\mathrm{~A} 3$ & $\mathbf{4}$ & $\mathbf{2}$ & $\mathbf{4}$ & $\mathbf{4}$ & $\mathbf{3}$ & $\mathbf{1}$ & $\mathbf{2}$ & $\mathbf{2}$ & $\mathbf{4}$ & $\mathbf{1}$ \\
\hline 4 & $\mathrm{~A} 4$ & $\mathbf{4}$ & $\mathbf{2}$ & $\mathbf{4}$ & $\mathbf{2}$ & $\mathbf{2}$ & $\mathbf{2}$ & $\mathbf{2}$ & $\mathbf{2}$ & $\mathbf{3}$ & $\mathbf{1}$ \\
\hline 5 & $\mathrm{~A} 5$ & $\mathbf{2}$ & $\mathbf{2}$ & $\mathbf{4}$ & $\mathbf{3}$ & $\mathbf{3}$ & $\mathbf{2}$ & $\mathbf{1}$ & $\mathbf{2}$ & $\mathbf{3}$ & $\mathbf{1}$ \\
\hline 6 & $\mathrm{~A} 6$ & $\mathbf{1}$ & $\mathbf{2}$ & $\mathbf{4}$ & $\mathbf{4}$ & $\mathbf{2}$ & $\mathbf{2}$ & $\mathbf{2}$ & $\mathbf{2}$ & $\mathbf{3}$ & $\mathbf{1}$ \\
\hline 7 & $\mathrm{~A} 7$ & $\mathbf{1}$ & $\mathbf{2}$ & $\mathbf{4}$ & $\mathbf{3}$ & $\mathbf{3}$ & $\mathbf{2}$ & $\mathbf{2}$ & $\mathbf{2}$ & $\mathbf{4}$ & $\mathbf{1}$ \\
\hline 8 & $\mathrm{~A} 8$ & $\mathbf{1}$ & $\mathbf{1}$ & $\mathbf{3}$ & $\mathbf{4}$ & $\mathbf{3}$ & $\mathbf{1}$ & $\mathbf{1}$ & $\mathbf{1}$ & $\mathbf{4}$ & $\mathbf{1}$ \\
\hline 9 & $\mathrm{~A} 9$ & $\mathbf{2}$ & $\mathbf{3}$ & $\mathbf{4}$ & $\mathbf{4}$ & $\mathbf{3}$ & $\mathbf{2}$ & $\mathbf{2}$ & $\mathbf{2}$ & $\mathbf{4}$ & $\mathbf{1}$ \\
\hline 10 & $\mathrm{~A} 10$ & $\mathbf{1}$ & $\mathbf{2}$ & $\mathbf{4}$ & $\mathbf{1}$ & $\mathbf{1}$ & $\mathbf{1}$ & $\mathbf{1}$ & $\mathbf{1}$ & $\mathbf{1}$ & $\mathbf{1}$ \\
\hline
\end{tabular}

Given a matrix $x(1,1)$ the divisor is obtained by:

$$
r_{i j}=\frac{x_{i j}}{\sqrt{\sum_{i=1}^{m} x_{i j}^{2}}}
$$

After determining the divider value, the normalized matrix is:

\section{TABLE V. NORMALIZATION MATRIX RESUlTS}

\begin{tabular}{|l|l|c|c|c|c|c|c|}
\hline No & Alternatif & \multicolumn{1}{|c|}{ C1 } & C2 & C3 & C4 & C5 & C6 \\
\hline 1 & A1 & .021739 & .06383 & .026144 & .033613 & .037975 & .064516 \\
\hline 2 & A2 & .021739 & .042553 & .026144 & .033613 & .050633 & .064516 \\
\hline 3 & A3 & .086957 & .042553 & .026144 & .033613 & .037975 & .032258 \\
\hline 4 & A4 & .086957 & .042553 & .026144 & .016807 & .025316 & .064516 \\
\hline 5 & A5 & .043478 & .042553 & .026144 & .02521 & .037975 & .064516 \\
\hline 6 & A6 & .021739 & .042553 & .026144 & .033613 & .025316 & .064516 \\
\hline 7 & A7 & .021739 & .042553 & .026144 & .02521 & .037975 & .064516 \\
\hline 8 & A8 & .021739 & .021277 & .019608 & .033613 & .037975 & .032258 \\
\hline 9 & A9 & .043478 & .06383 & .026144 & .033613 & .037975 & .064516 \\
\hline 10 & A10 & .021739 & .042553 & .026144 & .008403 & .012658 & .032258 \\
\hline
\end{tabular}

\begin{tabular}{|l|l|l|l|}
\hline C7 & C8 & C9 & C10 \\
\hline 0.055556 & 0.051282 & 0.032258 & 0.1 \\
\hline 0.083333 & 0.076923 & 0.032258 & 0.1 \\
\hline 0.055556 & 0.051282 & 0.032258 & 0.1 \\
\hline 0.055556 & 0.051282 & 0.024194 & 0.1 \\
\hline 0.027778 & 0.051282 & 0.024194 & 0.1 \\
\hline 0.055556 & 0.051282 & 0.024194 & 0.1 \\
\hline 0.055556 & 0.051282 & 0.032258 & 0.1 \\
\hline
\end{tabular}

\begin{tabular}{|l|l|l|l|}
\cline { 1 - 1 } 0.027778 & 0.025641 & 0.032258 & 0.1 \\
\hline 0.055556 & 0.051282 & 0.032258 & 0.1 \\
\hline 0.027778 & 0.025641 & 0.008065 & 0.1 \\
\hline
\end{tabular}

After getting the normalization matrix results, the results of the decision matrix table below can be obtained from the results of the normalized decision multiplied by the weight of Criteria Data with the formula

$$
y_{i j}=w_{i} \cdot r_{i j}
$$

The results of the criteria weights are as follows

\section{TABLE VI. NORMALIZATION WEIGTH}

\begin{tabular}{|l|l|l|l|l|l|l|}
\hline No & Alternatif & C1 & C2 & C3 & C4 & C5 \\
\hline 1 & A1 & 0.01087 & 0.031915 & 0.013072 & 0.016807 & 0.037975 \\
\hline 2 & A2 & 0.01087 & 0.021277 & 0.013072 & 0.016807 & 0.050633 \\
\hline 3 & A3 & 0.043478 & 0.021277 & 0.013072 & 0.016807 & 0.037975 \\
\hline 4 & A4 & 0.043478 & 0.021277 & 0.013072 & 0.008403 & 0.025316 \\
\hline 5 & A5 & 0.021739 & 0.021277 & 0.013072 & 0.012605 & 0.037975 \\
\hline 6 & A6 & 0.01087 & 0.021277 & 0.013072 & 0.016807 & 0.025316 \\
\hline 7 & A7 & 0.01087 & 0.021277 & 0.013072 & 0.012605 & 0.037975 \\
\hline 8 & A8 & 0.01087 & 0.010638 & 0.009804 & 0.016807 & 0.037975 \\
\hline 9 & A9 & 0.021739 & 0.031915 & 0.013072 & 0.016807 & 0.037975 \\
\hline 10 & A10 & 0.01087 & 0.021277 & 0.013072 & 0.004202 & 0.012658 \\
\hline
\end{tabular}

\begin{tabular}{|l|l|l|l|l|}
\hline C1 & C2 & C3 & C4 & C5 \\
\hline 0.01087 & 0.031915 & 0.013072 & 0.016807 & 0.037975 \\
\hline 0.01087 & 0.021277 & 0.013072 & 0.016807 & 0.050633 \\
\hline 0.043478 & 0.021277 & 0.013072 & 0.016807 & 0.037975 \\
\hline 0.043478 & 0.021277 & 0.013072 & 0.008403 & 0.025316 \\
\hline 0.021739 & 0.021277 & 0.013072 & 0.012605 & 0.037975 \\
\hline 0.01087 & 0.021277 & 0.013072 & 0.016807 & 0.025316 \\
\hline 0.01087 & 0.021277 & 0.013072 & 0.012605 & 0.037975 \\
\hline 0.01087 & 0.010638 & 0.009804 & 0.016807 & 0.037975 \\
\hline 0.021739 & 0.031915 & 0.013072 & 0.016807 & 0.037975 \\
\hline 0.01087 & 0.021277 & 0.013072 & 0.004202 & 0.012658 \\
\hline & & & &
\end{tabular}

After the normalized decision matrix is obtained, the results of the decision table below are the results of the normalized weight decision divided by the Maximal and Minimum normalized weights with the formula:

$$
\begin{aligned}
& A^{+}=\left(y_{1}^{+}, y_{2}^{+}, \ldots, y_{n}^{+}\right) \\
& A^{-}=\left(y_{1}^{-}, y_{2}^{-}, \ldots, y_{n}^{-}\right)
\end{aligned}
$$


Min Max Results Based on criteria

TABLE VII. ALLTERNATIVE $\boldsymbol{A}+\boldsymbol{D A N} \boldsymbol{A}$ -

\begin{tabular}{|l|l|}
\hline $\mathrm{A}+$ & $\mathrm{A}-$ \\
\hline 0.045027 & 0.049612 \\
\hline 0.0343 & 0.073038 \\
\hline 0.033798 & 0.055858 \\
\hline 0.040806 & 0.048788 \\
\hline 0.053909 & 0.039176 \\
\hline 0.051554 & 0.038186 \\
\hline 0.046457 & 0.045095 \\
\hline 0.07048 & 0.033601 \\
\hline 0.037903 & 0.050788 \\
\hline 0.07992 & 0.011129 \\
\hline
\end{tabular}

Determine the distance between each alternative's weighted value to the positive ideal solution and the negative ideal solution. To determine the distance between each alternative's weighted value to the positive ideal solution, use the following equation

$$
D_{i}^{+}=\sqrt{\sum_{i=1}^{n}\left(y_{i}^{+}-y_{i j}\right)^{2}}
$$

Next to calculate the distance between the weighted values of each alternative to the negative ideal solution, the following equation is used

$$
D_{i}^{-}=\sqrt{\sum_{i=1}^{n}\left(y_{i j}-y_{i}^{-}\right)^{2}}
$$

Alternative Results of D + and D-

TABLE VIII. ALLTERNATIVE $\boldsymbol{D}+\boldsymbol{D A N} \boldsymbol{D}$ -

\begin{tabular}{|l|l|}
\hline $\mathrm{D}+$ & $\mathrm{D}-$ \\
\hline 0.045027 & 0.049612 \\
\hline 0.0343 & 0.073038 \\
\hline 0.033798 & 0.055858 \\
\hline 0.040806 & 0.048788 \\
\hline 0.053909 & 0.039176 \\
\hline 0.051554 & 0.038186 \\
\hline 0.046457 & 0.045095 \\
\hline 0.07048 & 0.033601 \\
\hline 0.037903 & 0.050788 \\
\hline
\end{tabular}

\section{\begin{tabular}{l|l}
0.07992 & 0.011129 \\
\hline
\end{tabular}}

The final step is to calculate preferences for each alternative, namely the results of the alternative $\mathrm{D}+$ and $\mathrm{D}$ - added to the formula

$$
\begin{aligned}
& V_{i}=\frac{D_{i}^{-}}{D_{i}^{-}+D_{i}^{+}} \\
& V_{1}=\frac{0,049612}{0,04502+0,049612}=0.52422 \\
& V_{2}=\frac{0.04961}{0.04503+0.04961}=0.68045 \\
& V_{3}=\frac{0.07304}{0.03430+0.07304}=0.62303 \\
& V_{4}=\frac{0.05586}{0.03380+0.05586}=0.54454 \\
& V_{5}=\frac{0.04879}{0.04081+0.04879}=0.42086 \\
& V_{6}=\frac{0.03918}{0.05391+0.03918}=0.42552 \\
& V_{7}=\frac{0.03819}{0.05155+0.03819}=0.49256 \\
& V_{8}=\frac{0.04509}{0.07048+0.04509}=0.32284 \\
& V_{9}=\frac{0.05079}{0.03790+0.05079}=0.57264 \\
& V_{10}=\frac{0.01113}{0.07992+0.01113}=0.12223
\end{aligned}
$$

From the results of calculations carried out using the TOPSIS method obtained ranks of the best tertiary institutions in Medan City. The best results obtained are the highest value, A2

\section{CONCLUSION AND Suggestion}

The conclusions obtained from the results of this study are

1. With the application of the TOPSIS Method in Decision Support in the selection of the Best Private Universities in the City of Medan is able to provide optimal results based on predetermined criteria and weighting.

2. In giving the criteria weights and preference weights are very influential in the calculation results, therefore in giving the value of weights must be analyzed where the criteria whose weight is preferred. 


\section{ACKNOWLEDGMENT}

Thank you to the research institute and journal publication at the Harapan University of Medan, Mr. Ruswan Nurmadi, who facilitated the authors in the research conducted so that the publication of this publication. And thanks also to the Chancellor of Universitas Harapan Medan, Prof. Prof. Dr. Ritha F. Dalimunthe and thanks Dr. Rahmat Widia Sembiring who helped in the completion of the research the author did. And thanks to all those who cannot be mentioned one by one.

\section{REFERENCES}

Akmaludin, A., \& Badrul, M. (2019). Multi-criteria for Selection of SmartPhone Brands Product using AHP-TOPSIS Method. SinkrOn, 3(2), 154.

https://doi.org/10.33395/sinkron.v3i2.10069

Badrul, M., Rusdiansyah, R., \& Budihartanti, C. (2019). Application of Simple Additive Weighting Method for Determination of Toddler Nutrition Status. SinkrOn, 4(1), 19. https://doi.org/10.33395/sinkron.v4i1.10145

Chamid, A. A. (2016). Prioritas Kondisi Rumah, 7(2), 537-544.

Erik Kurniawan, Hindayati Mustafidah, A. S. (2015). Metode TOPSIS untuk Menentukan Penerimaan Mahasiswa Baru Pendidikan Dokter di Universitas Muhammadiyah Purwokerto ( TOPSIS Method to Determine New Students Admission at Medical School in University of, III(November), 201-206.

Firdaus, I. H., Abdillah, G., \& Renaldi, F. (2016). Sistem Pendukung Keputusan Penentuan
Karyawan Terbaik. Seminar Nasional Teknologi Informasi Dan Komunikasi 2016 (SENTIKA 2016), 2016(Sentika), 440-445. https://doi.org/10.1111/gcb.13996

Gustri Wahyuni, E., Khairunnisa, N., Abriyani, F., Muchlis, N. F., \& Ulfa, M. (2017). Sistem Pendukung Keputusan Pemilihan Asisten Terbaik Dengan Metode TOPSIS dan WP. Teknoin, 22(2), 93-100. https://doi.org/10.1016/j.marchem.2015.04.008

Herawatie, D., \& Wuryanto, E. (2017). Sistem Pendukung Keputusan Pemilihan Mahasiswa Berprestasi dengan Metode Fuzzy TOPSIS. Journal of Information System Engineering and Business Interligence, 3(2).

Kadarsah Suryadi, A. ramdhani. (2015). Sistem Pendukung Keputusan, (November), 10091024.

Kemenristekdikti. (2018). Kemenristekdikti Umumkan Peringkat 100 Besar Perguruan Tinggi Indonesia Non Vokasi Tahun 2018.

Muzakkir, I. (2017). Penerapan metode topsis untuk sistem pendukung keputusan penentuan keluarga miskin pada desa panca karsa ii. ILKOM Jurnal Ilmiah Volume 9 Nomor 3 Desember 2017, 9, 274-281.

Saleh, S., \& Informasi, F. S. (2016). ( STUDI KASUS YADIKA NATAR ), 16(02), 160-169.

Santiary, P. A. W., Ciptayani, P. I., Saptarini, N. G. A. P. H., \& I Ketut Swardika. (2018). Jurnal Pengertian Topsis, 5(5), 621-628. https://doi.org/10.25126/jtiik2018551120

Kusumadewi, S. H. (2006) 'Fuzzy Multi-Attribute Decision Making (Fuzzy MADM)', Graha Ilmu Yogyakarta. 